\title{
ARTICLE OPEN Revisiting the anchoring behavior in lithium-sulfur batteries: many-body effect on the suppression of shuttle effect
}

\author{
Min Fang ${ }^{1}$, Xinyi Liu ${ }^{1}$, Ji-Chang Ren ${ }^{1}$, Sha Yang ${ }^{1}$, Guirong $\mathrm{Su}^{1}$, Qin Fang ${ }^{1}$, Jianzhong $\mathrm{Lai}^{2}$, Shuang $\mathrm{Li}^{1 *}$ and Wei Liu $\mathbb{D}^{1 *}$
}

We apply the state-of-the-art many-body dispersion (MBD) method to study the anchoring behavior in lithium-sulfur (Li-S) batteries, which is closely related to the notorious "shuttle effect". Based on the experimental results of metal sulfides (FeS and $\mathrm{SnS}_{2}$ ), we find that the MBD method gives a more accurate prediction of anchoring mechanism compared with other van der Waals $(\mathrm{vdW})$ inclusive methods. We systematically investigate the anchoring mechanism of two prototypal anchoring materials- $\mathrm{Ti}_{2} \mathrm{CF}_{2}$ and doped-graphene systems. The many-body effect is found to play an important role on the reduction of anchoring behaviors, especially when the systems have large polarization and the vdW interactions predominate the anchoring behavior. Our work deepens the fundamental understanding of the anchoring mechanism, and provides a more accurate criterion for screening anchoring materials for suppressing the shuttle effect.

npj Computational Materials (2020)6:8; https://doi.org/10.1038/s41524-020-0273-1

\section{INTRODUCTION}

Lithium-sulfur batteries ( $\mathrm{Li}-\mathrm{S}$ ) fulfill the urgent demands on the storage and transport of renewable energy, owing to their overwhelming theoretical energy density and relatively low cost. $^{1-7}$ However, one of the fatal issues that restricts the commercialization of $\mathrm{Li}-\mathrm{S}$ batteries is the notorious "shuttle effect", which stems from the dissolution and the endless transport/shuttle of lithium polysulfides (LiPSs) between cathode and anode during the redox process. These phenomena greatly reduce the utilization of sulfur and Coulomb efficiency, causing the self-discharge behavior and the rapid decrease of battery capacity. ${ }^{8}$ For the practical applications of Li-S batteries, the shuttle effect has to be greatly suppressed in liquid electrolyte. ${ }^{9}$

An effective strategy for overcoming the shuttle effect is to introduce anchoring materials in cathode to trap LiPSs. Conductive mesoporous carbon framework was first used to encapsulate the sulfur cathode. ${ }^{10}$ Subsequently, functionalized carbon materials were developed to enhance the polarity of carbon materials. ${ }^{11}$ Recently, two-dimensional (2D) materials like polar metallic oxides, ${ }^{12,13}$ transition-metal sulfides, ${ }^{14}$ and metal nitrides, ${ }^{15}$ were utilized to alleviate the shuttle effect, but their applications are limited by their bad conductivity. Encouragingly, 2D transition-metal carbides, carbonitrides, and nitrides (known as MXenes) ${ }^{16}$ have been demonstrated to greatly promote the electrochemical performance for energy storage devices. ${ }^{17-20}$ Because of their prominent advantages, such as metallic conductivity, a plastic layer structure, and the hydrophilic nature of its functionalized surface, ${ }^{21}$ MXenes have been intensively investigated as anchoring materials in Li-S batteris. ${ }^{22-24}$

The accurate prediction of binding energies between anchoring materials, electrolytes, and LiPSs is a prerequisite for uncovering the anchoring mechanism and design cathode in Li-S batteries. ${ }^{25-27}$ In this contribution, we apply the many-body dispersion (MBD) method to investigate two prototypal anchoring materials and find that the many-body effect significantly affects the binding energies of LiPSs on $\mathrm{Ti}_{2} \mathrm{CF}_{2}$. In this system the larger many-body effect originates from the large polarization and the domination of van der Waals (vdW) forces. In contrast, for N-doped graphene (NG) systems, the contribution of the many-body effect for binding strength is only $2 \%$, since the chemical bonding predominates the whole process of lithiation. Our work paves the way for more reliably screening anchoring materials to control the shuttle effect and to design high-performance cathode materials for Li-S batteries.

\section{RESULTS}

Performance of FeS and $\mathrm{SnS}_{2}$ for trapping $\mathrm{Li}_{2} \mathrm{~S}_{6}$

Zhou et al. $^{28}$ have experimentally investigated the anchoring behavior of metal sulfides for trapping $\mathrm{Li}_{2} \mathrm{~S}_{6}$ by comparative tests (different metal sulfides with equivalent total surface area were added into originally yellow-colored 1, 2-dimethoxyethane/1, 3dioxolane (DME/DOL) solution with $\mathrm{Li}_{2} \mathrm{~S}_{6}$ ). According to the discoloration of solution, they found that the metal sulfides have different adsorption capability for $\mathrm{Li}_{2} \mathrm{~S}_{6}$ and several of them completely suppressed the shuttle effect. The competition between anchoring materials and electrolytes for trapping soluble LiPSs has been concerned to evaluate anchoring materials. ${ }^{29,30}$ However, in their density functional theory (DFT) calculation the anchoring factor $\Delta E\left(\Delta E=E_{\mathrm{AM}}^{\mathrm{b}}-E_{\text {so }}^{\mathrm{b}}\right)$ was not considered. Here $E_{\mathrm{AM}}^{\mathrm{b}}$ is the binding energies of LiPSs absorbed on anchoring materials, and $E_{\text {so }}^{\mathrm{b}}$ denotes the binding energies of LiPSs with electrolyte molecules. The positive $\Delta E$ indicates that the interaction between polysulfides and anchoring materials is stronger than polysulfides with electrolyte molecules; the negative $\Delta E$ means that polysulfides tend to dissolve into electrolyte rather than absorb on anchoring materials.

To compare theoretical prediction from different vdW methods, we apply a criterion $(\Delta E)$ to evaluate the performance of FeS and $\mathrm{SnS}_{2}$ for trapping $\mathrm{Li}_{2} \mathrm{~S}_{6}$ and compare with experimental observation. ${ }^{28}$ The optimized configurations of $\mathrm{Li}_{2} \mathrm{~S}_{6}$ absorbed on FeS and $\mathrm{SnS}_{2}$, and its binding with DME/DOL molecule are shown in Supplementary Fig. 1. Recently, Brandenburg et al. ${ }^{31}$ reported that the MBD method performs better than most other approaches,

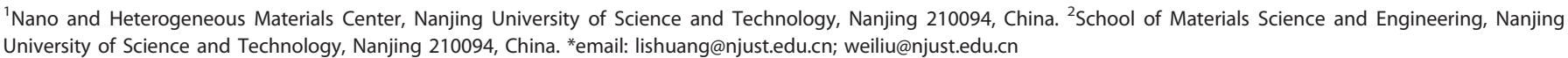


Table 1. The binding energies (eV) between $\mathrm{Li}_{2} \mathrm{~S}_{6}$ and anchoring materials ( $\mathrm{FeS}$ and $\mathrm{SnS}_{2}$ ) as well as electrolyte molecules (DME and $\mathrm{DOL}$ ) from several DFT methods, the anchoring factor $\Delta \mathrm{E}$ are also listed.

\begin{tabular}{|c|c|c|c|c|c|c|}
\hline $\mathrm{Li}_{2} \mathrm{~S}_{6}$ & $\mathrm{PBE}+\mathrm{MBD}$ & $\mathrm{PBE}+\mathrm{vdW}$ surf & $\mathrm{PBE}+\mathrm{D} 2$ & $\mathrm{PBE}+\mathrm{D} 3$ & vdW-DF2 & optB86b-vdW \\
\hline $\mathrm{SnS}_{2}$ & 0.87 & 1.06 & 0.79 & 0.96 & 1.27 & 0.94 \\
\hline DOL & 0.82 & 0.77 & 0.84 & 0.78 & 0.91 & 0.74 \\
\hline$\Delta \mathrm{E}(\mathrm{FeS}-\mathrm{DME})$ & -0.10 & 0.48 & -0.06 & 0.18 & 0.43 & 0.19 \\
\hline$\Delta \mathrm{E}\left(\mathrm{SnS}_{2}-\mathrm{DOL}\right)$ & 0.05 & 0.29 & -0.05 & 0.18 & 0.36 & 0.20 \\
\hline
\end{tabular}

such as DMC and $\operatorname{CCSD}(T)$, for the fundamental system. Meanwhile, it has been demonstrated that it is essential to include the many-body effect in adsorption systems for the evaluation of binding energies. ${ }^{32-34}$ In this context, here we apply the MBD method to predict the interaction between $\mathrm{Li}_{2} \mathrm{~S}_{6}$ and $\mathrm{FeS} / \mathrm{SnS} \mathrm{S}_{2}$. From the results of PBE + MBD method in Table 1, $\triangle E$ (FeS-DME) and $\triangle E\left(\mathrm{SnS}_{2}-\mathrm{DME}\right)$ are negative values $(-0.10 \mathrm{eV}$ and $-0.07 \mathrm{eV})$, $\Delta E(\mathrm{FeS}-\mathrm{DOL})$ and $\Delta E\left(\mathrm{SnS}_{2}-\mathrm{DOL}\right)$ are positive values $(0.02 \mathrm{eV}$ and $0.05 \mathrm{eV}$ ). We predict that a part of $\mathrm{Li}_{2} \mathrm{~S}_{6}$ species will be anchored on the surface of $\mathrm{FeS}$ and $\mathrm{SnS}_{2}$ and the rest of $\mathrm{Li}_{2} \mathrm{~S}_{6}$ still binds with DME molecules. In other words, the yellow DME/DOL solution with $\mathrm{Li}_{2} \mathrm{~S}_{6}$ just has an incompletely discoloration when FeS and $\mathrm{SnS}_{2}$ are added as anchoring materials. This conclusion is fully compatible with the experimental observation.

However, from the PBE + D2 method, the $\Delta E$ (FeS-DOL) is $0.04 \mathrm{eV}$ and other $\Delta E$ values are negative, which suggests that $\mathrm{SnS}_{2}$ cannot trap $\mathrm{Li}_{2} \mathrm{~S}_{6}$ species efficiently. Therefore, the yellow DME/DOL solution with $\mathrm{Li}_{2} \mathrm{~S}_{6}$ should keep the color invariable when $\mathrm{SnS}_{2}$ is used as anchoring materials. From the PBE + $\mathrm{vdW}^{\text {surf }}, \mathrm{PBE}+\mathrm{D} 3, \mathrm{vdW}-\mathrm{DF} 2$ and optB86b-vdW methods, all $\Delta E$ are positive values, implying that $\mathrm{Li}_{2} \mathrm{~S}_{6}$ is absorbed on $\mathrm{FeS}$ and $\mathrm{SnS}_{2}$ firmly and scarcely binds with DME/DOL molecules. Thus, the yellow DME/DOL solution with $\mathrm{Li}_{2} \mathrm{~S}_{6}$ should fade completely. Nevertheless, the experiment clearly showed that when FeS and $\mathrm{SnS}_{2}$ are added as anchoring materials, the originally yellowcolored polysulfide solution becomes lighter in color rather than become colorless in experimental observation. Therefore, the conclusion from those computational methods is inconsistent with experimental observation.

\section{Performance of $\mathrm{Ti}_{2} \mathrm{CF}_{2}$ and NG for trapping LiPSs}

According to the above analysis, the MBD method is more accurate than other methods to describe the interaction between LiPSs and metal sulfides. However, many studies also got reasonable conclusions of the anchoring materials for suppression of the shuttle effect by pairwise-based methods. ${ }^{26,30,35}$ Therefore, to attain an in-depth understanding of the many-body effect on binding strength of LiPSs with anchoring materials, we selected $\mathrm{Ti}_{2} \mathrm{CF}_{2}$ and $\mathrm{NG}$ as platforms and investigated their intrinsic different anchoring mechanisms. $\mathrm{Ti}_{2} \mathrm{C}$-based materials are the lightest Mxenes and atom-multilayered 2D materials, NG is a typical heteroatom-doped anchoring material with one-atomiclayer structure. As for $\mathrm{Ti}_{2} \mathrm{CF}_{2}$, we note that the F-termination has different adsorption sites on $\mathrm{Ti}_{2} \mathrm{C}$ surface ${ }^{36,37}$ and the HCP site is chosen in our work because of the stronger binding strength of LiPSs (Supplementary Fig. 2 and Supplementary Table 1). Firstly, the configurations of soluble LiPSs with electrolyte molecules are investigated. Notably, the real electrolyte system is very complicated, which usually consists of solvents, lithium salts, and (a)

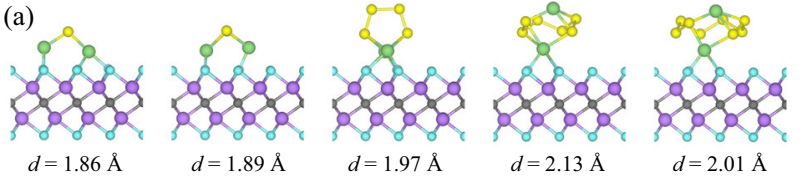

(b)

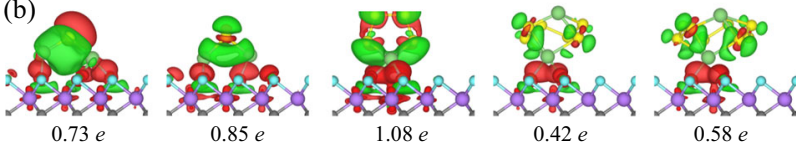

Fig. 1 The adsorption configurations and charge transfers of LiPSs adsorbed on $\mathbf{T i}_{\mathbf{2}} \mathbf{C F}_{\mathbf{2}}$. a Side views of the most stable configurations of $\mathrm{Li}_{2} \mathrm{~S}_{\mathrm{n}}(n=1,2,4,6,8)$ anchoring on $\mathrm{Ti}_{2} \mathrm{CF}_{2}$. The shortest length of $\mathrm{Li}-\mathrm{S}$ bond is also labeled. b Charge transfers between LiPSs and $\mathrm{Ti}_{2} \mathrm{CF}_{2}$. Here, red (green) is the spatial regions gain (loss) in charge. In all configurations LiPSs lose charge and $\mathrm{Ti}_{2} \mathrm{CF}_{2}$ obtain charge. The value of charge transfer is labeled. The purple, gray, sky blue, yellow, and green spheres represent the $\mathrm{Ti}, \mathrm{C}$, $\mathrm{F}, \mathrm{S}$, and Li atoms, respectively.

additives. $^{38}$ Nevertheless, the major component causing the shuttle effect is solvent molecules in electrolyte, which competes with anchoring materials and introduces solution of high-order LiPSs. As such, in the present work, and the same in previous work, $^{29,30}$ we used DME and DOL molecules to explain the interaction between LiPSs and electrolyte. We considered that one LiPSs cluster interacts with one or two DME (DOL) molecules (Supplementary Fig. 3). From Supplementary Table 2 we find that the $E_{\text {so }}^{\mathrm{b}}$ are very similar whatever soluble LiPSs binding with one or two DME (DOL) molecules from the vdW ${ }^{\text {surf }}$ and MBD methods. For systematically examining the $E_{\mathrm{AM}}^{\mathrm{b}}$ between LiPSs and $\mathrm{Ti}_{2} \mathrm{CF}_{2}$ / $\mathrm{NG}$, various initial adsorption configurations of $\mathrm{Li}_{2} \mathrm{~S}_{n}(n=1,2,4,6$, and 8) clusters are considered to find the optimized structures. The most stable structures with the lowest energy are shown in Fig. 1a. Comparing with $\mathrm{Li}_{2} \mathrm{~S}_{6}$ and $\mathrm{Li}_{2} \mathrm{~S}_{8}, \mathrm{Li}_{2} \mathrm{~S}_{1} \mathrm{Li}_{2} \mathrm{~S}_{2}$, and $\mathrm{Li}_{2} \mathrm{~S}_{4}$ prefer to adsorb vertically on $\mathrm{Ti}_{2} \mathrm{CF}_{2}$, with two $\mathrm{Li}$ atoms binding with $\mathrm{Ti}_{2} \mathrm{CF}_{2}$. We also search the most stable configurations in NG systems (Supplementary Fig. 4). The calculated $E_{A M}^{b}$ of most stable configurations from $\mathrm{PBE}+\mathrm{vdW}$ surf, $\mathrm{PBE}+\mathrm{MBD}$, and other methods at different lithiation stages are shown in Table 2, Fig. 2a, b, and Supplementary Table 3.

Figure $2 \mathrm{a}$ shows that the $\mathrm{PBE}+\mathrm{MBD}$ method reduces the $E_{\mathrm{AM}}^{\mathrm{b}}$ over $20 \%$ compared to the $\mathrm{PBE}+\mathrm{vdW}^{\text {surf }}$ method at high-order lithiation stages for $\mathrm{Ti}_{2} \mathrm{CF}_{2}$ systems. In contrast, the binding energies for the NG systems from the two methods are almost identical (Fig. 2b). To assess the contribution of MBD to the $E_{A M}^{b}$, we determine the ratio of $\mathrm{MBD}$ contribution $\left(R_{\mathrm{MBD}}\right)$ as follows,

$R_{\mathrm{MBD}}=\left(E_{\mathrm{vdW}}^{\mathrm{b}}-E_{\mathrm{MBD}}^{\mathrm{b}}\right) / E_{\mathrm{MBD}}^{\mathrm{b}}$ 
Table 2. Computed binding energies (eV) between LiPSs and $\mathrm{Ti}_{2} \mathrm{CF}_{2} / \mathrm{NG}$ from the $\mathrm{PBE}+\mathrm{vdW}$ surf and PBE $+\mathrm{MBD}$ methods.

\begin{tabular}{|c|c|c|c|c|c|c|c|c|c|c|}
\hline & \multicolumn{5}{|l|}{$\mathrm{Ti}_{2} \mathrm{CF}_{2}$} & \multicolumn{5}{|l|}{ NG } \\
\hline $\mathrm{Li}_{2} \mathrm{~S}$ & 2.36 & 2.02 & 16.8 & 53.5 & - & 2.76 & 2.80 & 1.4 & 17.9 & - \\
\hline $\mathrm{Li}_{2} \mathrm{~S}_{4}$ & 1.61 & 1.24 & 29.8 & 99.2 & 0.34 & 2.33 & 2.33 & 0.0 & 34.8 & 1.43 \\
\hline $\mathrm{Li}_{2} \mathrm{~S}_{6}$ & 1.28 & 0.96 & 33.3 & 110.4 & 0.02 & 2.37 & 2.34 & 1.3 & 41.9 & 1.40 \\
\hline
\end{tabular}
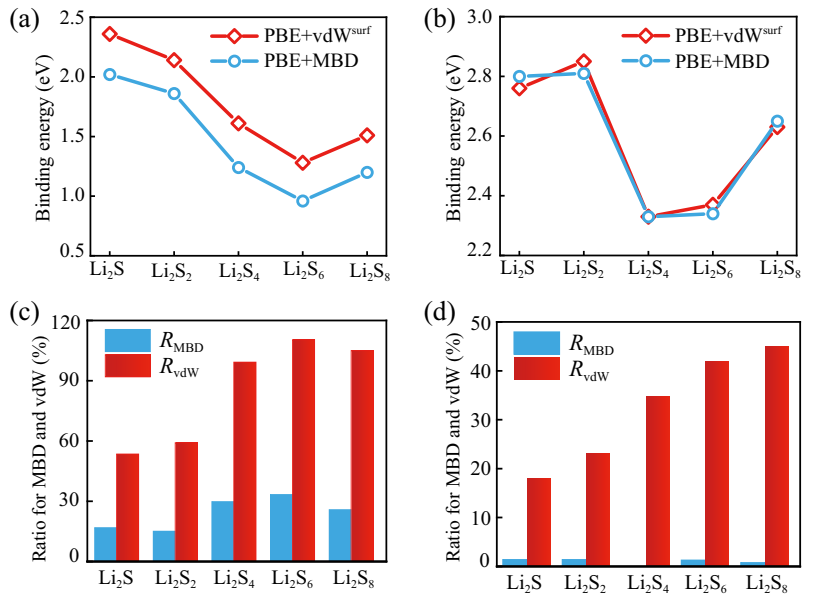

Fig. 2 The binding energies and ratio for many-body effect and $\mathbf{v d W}$ interaction of $\mathrm{Ti}_{2} \mathbf{C F}_{2}$ and $\mathbf{N G}$ systems. Computed binding energies for $\mathbf{a}$ LiPSs on $\mathrm{Ti}_{2} \mathrm{CF}_{2}$ and $\mathbf{b}$ LiPSs on NG with the PBE + $v^{\text {vdW }} W^{\text {surf }}$ and PBE + MBD methods. The ratio for many-body effect $\left(R_{\mathrm{MBD}}\right)$ and vdW interaction $\left(R_{\mathrm{vdW}}\right)$ of binding energies in $(\mathbf{c}) \mathrm{Ti}_{2} \mathrm{CF}_{2}$ systems and d NG systems.

where $E_{\mathrm{vdW}}^{\mathrm{b}}$ and $E_{\mathrm{MBD}}^{\mathrm{b}}$ denote the binding energies of $\mathrm{Ti}_{2} \mathrm{CF}_{2}$ (NG) from the $\mathrm{PBE}+$ vdW $\mathrm{W}^{\text {surf }}$ and $\mathrm{PBD}+\mathrm{MBD}$ methods, respectively. The magnitudes of $R_{\mathrm{MBD}}$ for LiPSs absorbed on $\mathrm{Ti}_{2} \mathrm{CF}_{2}$ and NG are shown in Fig. $2 \mathrm{c}$, d. From Table 2, when $\mathrm{Ti}_{2} \mathrm{CF}_{2}$ are selected as anchoring materials, we find that $R_{\mathrm{MBD}}$ varies from $16.8 \%$ to $33.3 \%$ with the delithiation of LiPSs. The many-body effect affects $\mathrm{Li}_{2} \mathrm{~S}_{6}$ $\mathrm{Ti}_{2} \mathrm{CF}_{2}$ system largely, which has weakened the binding strength by $33.3 \%$ and may lead to the dissolution of $\mathrm{Li}_{2} \mathrm{~S}_{6}$ due to the small $\triangle E(0.02 \mathrm{eV})$. While for the NG system, we find that the $R_{\mathrm{MBD}}$ (range from 0 to $1.4 \%$ ) is slight and the many-body effect could be ignored.

To attain an in-depth understanding why the MBD method reduces the binding strength of LiPSs with anchoring materials, we divide the $E^{\mathrm{b}}{ }_{\mathrm{AM}}$ into two aspects: the vdW part corresponds to the physical interaction, and the PBE part (termed as $E_{\mathrm{PBE}}^{\mathrm{b}}$ ) corresponds to the chemical interaction using pairwise-based method vdW $W^{\text {surf }}$, because the MBD effect mainly contributes to the part of physical interaction. To clarify how these two aspects affect the anchoring behavior, we define the ratio of the vdW interaction $\left(R_{\mathrm{vdW}}\right)$ as follows,

$R_{\mathrm{vdW}}=\left(E_{\mathrm{vdW}}^{\mathrm{b}}-E_{\mathrm{PBE}}^{\mathrm{b}}\right) / E_{\mathrm{vdW}}^{\mathrm{b}}$

In Eq. (2), $E_{\mathrm{vdW}}^{\mathrm{b}}$ denotes $E_{\mathrm{AM}}^{\mathrm{b}}$ from the pairwise $\mathrm{PBE}+\mathrm{vdW}$ surf method. The ratio of vdW contribution to $E_{\mathrm{AM}}^{\mathrm{b}}$ for $\mathrm{Ti}_{2} \mathrm{CF}_{2}$ systems is shown in Fig. $2 c$, the vdW interaction shows different weights towards different lithiation stages. During the delithiation from
$\mathrm{Li}_{2} \mathrm{~S}$ to $\mathrm{Li}_{2} \mathrm{~S}_{8}$, the Li-F bond length increases from 1.86 to $2.01 \AA$, suggesting that the chemical interaction is gradually weakened. The bond length is 1.86 and $1.88 \AA$ for $\mathrm{Li}_{2} \mathrm{~S}$ and $\mathrm{Li}_{2} \mathrm{~S}_{2}$ absorbed on $\mathrm{Ti}_{2} \mathrm{CF}_{2}$ (within the distances of covalent bonds), and the $R_{\mathrm{vdw}}$ is $53 \%$ and $59 \%$, respectively. This suggests that both chemical interaction and vdW interactions play important roles in these two adsorption systems. However, the physical interaction dominates the $E_{\mathrm{AM}}^{\mathrm{b}}$ of high-order $\mathrm{Li}_{2} \mathrm{~S}_{\mathrm{n}}$ species $(n=4,6,8)$ absorbed on $\mathrm{Ti}_{2} \mathrm{CF}_{2}$ (the $R_{\mathrm{vdw}}$ nearly $100 \%$ ). In contrast, although the $R_{\mathrm{vdw}}$ for the NG systems increases with the increasing size of LiPSs, the $R_{\mathrm{vdW}}$ of NG systems (ranges from $18 \%$ to $45 \%$ ) is smaller than those of $\mathrm{Ti}_{2} \mathrm{CF}_{2}$ systems especially for high-order LiPSs, so the chemical interaction (the bond length of Li-N ranges from $1.86 \AA$ to $1.90 \AA$ ) dominates the whole process of lithiation (Fig. $2 d$ ).

Static polarizability of adsorption systems and charge transfer Comparing with pairwise vdW-correction methods, the MBD method computes the correlation energy of long-range electrostatic screening through the coupled harmonic oscillator model Hamiltonian, and considers dipolar vdW interactions to all orders in perturbation theory. ${ }^{39}$ The reduction of $E_{\mathrm{AM}}^{\mathrm{b}}$ from the $\mathrm{PBE}+$ MBD method must come from the collective inclusion of the longrange electrostatic screening. Polarizability is central to the description of the long-range electron correlation, which indicates the willingness of a material to respond under the influence of a perturbation of the electric field. It is known that five atomic layers of $\mathrm{Ti}_{2} \mathrm{CF}_{2}$ are stacked along the $c$-axis direction, middle $\mathrm{Ti}$ atoms experience the dynamic electric field created by the upper and lower atoms, which gives rise to polarization effects. ${ }^{40}$ To provide a quantitative description of the polarizability, we define the changes in static polarizability of the adsorption systems $\Delta a$ by

$\Delta a=\Delta a_{\text {total }}-\Delta a_{\text {AM }}-\Delta a_{\text {LiPSs }}$

where $\Delta a_{\text {total }}, \Delta a_{\mathrm{AM}}$, and $\Delta a_{\mathrm{LiPSs}}$ represent the screened static polarizabilities of the adsorption systems, the anchoring materials and the free-standing LiPSs, respectively. Considering the similar polarizabilities along $x$ and $y$ coordinates, we present the $\Delta a$ along $x$ and $z$ directions, termed as $\Delta a_{\|}$and $\Delta a_{\perp}$, which are parallel and perpendicular to the surface of $2 \mathrm{D}$ anchoring materials, respectively. The calculated $\Delta a\left(\Delta \mathrm{a}_{\|}\right.$and $\left.\Delta a_{\perp}\right)$ are listed in Table 3 . We find that for $\mathrm{Ti}_{2} \mathrm{CF}_{2}$ systems, contrary to the magnitude of $\Delta a_{\|}$that varies slightly, the magnitudes of $\Delta a_{\perp}$ are very different from the delithiation of LiPSs. We find that $\Delta a_{\perp}$ are positive for $\mathrm{Ti}_{2} \mathrm{CF}_{2}$ systems, which means that the adsorption of LiPSs increases the $\Delta a_{\perp}$ of the systems. However, the values of $\Delta a_{\perp}$ in $\mathrm{N}$-doped graphene adsorption systems are negative, indicating that the $\Delta a_{\perp}$ are decreased after LiPSs adsorbed on $\mathrm{N}$-doped graphene.

We notice that the magnitudes of $\Delta a_{\perp}$ of $\mathrm{Li}_{2} \mathrm{~S}_{6}$ and $\mathrm{Li}_{2} \mathrm{~S}_{8}$ absorbed on $\mathrm{Ti}_{2} \mathrm{CF}_{2}$ decrease to 10 bohr $^{3}$. The decreasing values of $\Delta a_{\perp}$ suggest the weakening of the polarization along 
Table 3. The total static polarizability $(\Delta a)$, and the $\Delta a$ along $x\left(\Delta a_{\|}\right)$ and $z\left(\Delta a_{\perp}\right)$ directions of $\mathrm{Ti}_{2} \mathrm{CF}_{2}$ adsorption configurations.

\begin{tabular}{llllll}
\hline $\mathrm{Ti}_{2} \mathrm{CF}_{2}$ & $\mathrm{Li}_{2} \mathrm{~S}$ & $\mathrm{Li}_{2} \mathrm{~S}_{2}$ & $\mathrm{Li}_{2} \mathrm{~S}_{4}$ & $\mathrm{Li}_{2} \mathrm{~S}_{6}$ & $\mathrm{Li}_{2} \mathrm{~S}_{8}$ \\
\hline$\Delta a\left(\mathrm{bohr}^{3}\right)$ & 33.7 & 41.3 & 37.2 & 22.7 & 22.2 \\
$\Delta a_{\|}\left(\mathrm{bohr}^{3}\right)$ & 52.1 & 51.2 & 54.2 & 51.4 & 48.3 \\
$\Delta a_{\perp}\left(\mathrm{bohr}^{3}\right)$ & 32.7 & 52.8 & 48.9 & 10.5 & 9.6 \\
$Q(\mathrm{e})$ & 0.73 & 0.85 & 1.08 & 0.42 & 0.58 \\
$\mathrm{NG}$ & $\mathrm{Li}_{2} \mathrm{~S}$ & $\mathrm{Li}_{2} \mathrm{~S}_{2}$ & $\mathrm{Li}_{2} \mathrm{~S}_{4}$ & $\mathrm{Li}_{2} \mathrm{~S}_{6}$ & $\mathrm{Li}_{2} \mathrm{~S}_{8}$ \\
$\Delta \alpha\left(\mathrm{bohr}^{3}\right)$ & 4.3 & 5.7 & 12.0 & 11.9 & 11.0 \\
$\Delta a_{\perp}\left(\mathrm{bohr}^{3}\right)$ & -7.4 & -9.2 & -20.3 & -12.1 & -14.7 \\
$\mathrm{Q}(\mathrm{e})$ & 0.67 & 0.75 & 0.55 & 0.36 & 0.38 \\
\hline
\end{tabular}

Summary of the static polarizability and charge transfer in $\mathrm{Ti}_{2} \mathrm{CF}_{2}$ and $\mathrm{NG}$ systems

The charge transfer $(\mathrm{Q})$ between LiPSs and $\mathrm{Ti}_{2} \mathrm{CF}_{2}(\mathrm{NG})$ are also shown in the table (a)

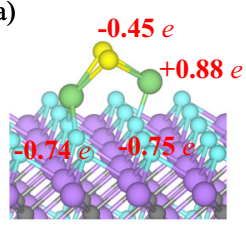

(d)

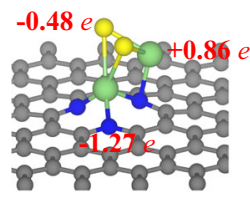

(b)

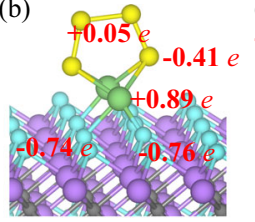

(e)

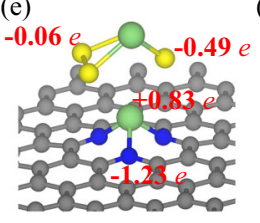

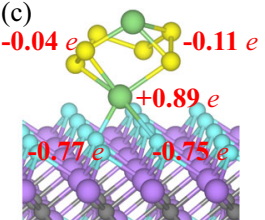

(f)

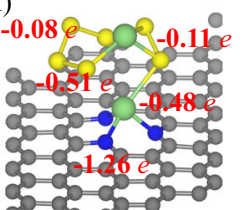

Fig. 3 Charge distribution analysis of LiPSs adsorbed on $\mathrm{Ti}_{2} \mathrm{CF}_{2}$ and NG. a-c The charge transfers $(e)$ of $\mathrm{S}$ and $\mathrm{Li}$ atoms in $\mathrm{Li}_{2} \mathrm{~S}_{2}, \mathrm{Li}_{2} \mathrm{~S}_{4}$ and $\mathrm{Li}_{2} \mathrm{~S}_{8}$ and adjacent $\mathrm{F}$ atoms in $\mathrm{Ti}_{2} \mathrm{CF}_{2}$ adsorption configurations. d-f The charge transfers between $\mathrm{S} / \mathrm{Li}$ atoms and adjacent $\mathrm{N}$ atoms in NG adsorption configurations.

perpendicular direction. The characteristics of anisotropic polarizability can be simply estimated by atomic polarizability matrix, which exhibits both spatial distributions and orientations of the polarizability in LiPSs cluster. For $\mathrm{Li}_{2} \mathrm{~S}_{4}$ adsorbed on $\mathrm{Ti}_{2} \mathrm{CF}_{2}$ in Supplementary Fig. 5, the polarizability matrixes show different anisotropic characteristics and the xy-orientational polarizability is much larger than that of $\mathrm{Li}_{2} \mathrm{~S}_{6}$ and $\mathrm{Li}_{2} \mathrm{~S}_{8}$, consistent with the optimized adsorption structures. Figure $2 \mathrm{a}$ shows that the $\mathrm{Li}_{2} \mathrm{~S}$, $\mathrm{Li}_{2} \mathrm{~S}_{2}$, and $\mathrm{Li}_{2} \mathrm{~S}_{4}$ species "stand" on the surface with two $\mathrm{Li}$ atoms parallel to the surface of $\mathrm{Ti}_{2} \mathrm{CF}_{2}$, along with a charge transfer of 0.73-1.08 e. For $\mathrm{Li}_{2} \mathrm{~S}_{6}$ and $\mathrm{Li}_{2} \mathrm{~S}_{8}$, however, the configurations transform to "lying-in-plane" configuration, with only one $\mathrm{Li}$ atom binding with $\mathrm{Ti}_{2} \mathrm{CF}_{2}$, and a decreased charge transfer $(0.42 \mathrm{e}$ for $\mathrm{Li}_{2} \mathrm{~S}_{6}$ and 0.58 e for $\mathrm{Li}_{2} \mathrm{~S}_{8}$ ).

In light of the charge distribution analysis ${ }^{41}$ shown in Fig. 3, we find that all $\mathrm{F}$ atoms in the F-terminated surface have a negative charge of 0.75 e. As for NG, N atoms obtain a large charge of $1.2 e$, but other $\mathrm{C}$ atoms are nearly electroneutral. Since the vdW forces arise from the electrostatic interactions between the constantly fluctuating electron clouds, the spontaneous polarization in $\mathrm{Ti}_{2} \mathrm{CF}_{2}$ make the polarizability sensitive to electron exchange. The above analysis shows that reduction of electron transfer is probably the major reason that causes the sharply reduction of the $\Delta a_{\perp}$. Meanwhile, from the density of states (DOS) of $\mathrm{NG}$ and $\mathrm{Ti}_{2} \mathrm{CF}_{2}$ (Supplementary Fig. 6) we find that they maintained its metallic properties after adsorption of $\mathrm{Li}_{2} \mathrm{~S}_{6}$, implying that they can supply electrons directly to the redox reaction of the anchored LiPSs. To

further understand the many-body effect in MXene systems, we calculated the anchoring behavior of $\mathrm{Ti}_{3} \mathrm{C}_{2} \mathrm{~F}_{2}$ (Supplementary Table 4), which was also applied as an anchoring material in $\mathrm{Li}-\mathrm{S}$ batteries. ${ }^{42}$ Compared with five atomic-layer $\mathrm{Ti}_{2} \mathrm{CF}_{2}$, we find that the polarizability perpendicular to plane $\left(\Delta a_{\perp}\right)$ and the ratio of MBD contribution $\left(R_{\mathrm{MBD}}\right)$ are both enhanced with the increasing thickness of anchoring materials.

From the above analysis, we find that $\mathrm{Ti}_{2} \mathrm{CF}_{2}$ systems has large polarization (22.2-41.3 bohr $^{3}$ ) and adsorption of LiPSs is dominated by the vdW interactions, which generates stronger manybody effect (large $R_{\mathrm{MBD}}$ ). We also investigate other 2D materials such as $\mathrm{TiS}_{2}, \mathrm{Ti}_{2} \mathrm{CO}_{2}{ }^{43,44}$ and $\mathrm{Ti}_{3} \mathrm{C}_{2} \mathrm{~F}_{2}{ }^{20,28}$ and the binding energies from two methods (PBE + vdW $^{\text {surf }}$ and PBE + MBD) are listed in Supplementary Table 5. We find that the many-body effect also exists in these systems. Therefore, for this type of anchoring materials with large $R_{\mathrm{MBD}}$, pairwise-vdW methods tend to overestimate the anchoring performance $(\Delta E)$ even get an inverse conclusion of anchoring materials. Conversely, chemical bonding plays an important role during the whole process of lithiation in NG systems, and NG has a small polarization (4.3-12 bohr ${ }^{3}$ ). As such, $R_{\mathrm{MBD}}$ is very small and pairwise-vdW and MBD method have the same prediction about $\Delta E$.

In Li-S batteries, the adsorption of LiPSs on loading materials or solvent molecules is a process of competition, thus positive $\Delta E$ is the prerequisite for the anchoring materials. ${ }^{4}$ It is clearly seen that NG has a large $\Delta E$, which agrees with the experimental results that NG can effectively anchor lithium polysulfides. However, the limited number of adsorption sites discourages high energy density of sulfur cathodes. In addition, the $\Delta E$ of $\mathrm{Li}_{2} \mathrm{~S}_{6}$ is as small as $0.02 \mathrm{eV}$, which demonstrates that $\mathrm{Ti}_{2} \mathrm{CF}_{2}$ would not effectively trap $\mathrm{Li}_{2} \mathrm{~S}_{6}$ species, and the desorption and dissolution of $\mathrm{Li}_{2} \mathrm{~S}_{6}$ species has a high probability to take place during lithiation.

\section{DISCUSSION}

We find the many-body dispersion interaction has a substantial impact on anchoring mechanism through binding energies of adsorption systems, especially for $\mathrm{Ti}_{2} \mathrm{CF}_{2}$ with strong polarization. According to the anchoring factor $\triangle E$ from the $\mathrm{PBE}+\mathrm{MBD}$ method, we theoretically predict that $\mathrm{Li}_{2} \mathrm{~S}_{6}$ partly dissolves into electrolytes solution and the DME/DOL solution with $\mathrm{Li}_{2} \mathrm{~S}_{6}$ discolors incompletely when FeS and $\mathrm{SnS}_{2}$ as anchoring materials, which agree well with the experiment. We demonstrated that the MBD approach can accurate predict the anisotropic polarizability and the anchoring behavior in the $\mathrm{Li}-\mathrm{S}$ batteries. By systematically investigating the many-body effect on $\mathrm{Ti}_{2} \mathrm{CF}_{2}$ and NG systems, we found that the PBE + MBD method reduces the $E_{A M}^{b}$ over $20 \%$, as compared to the $\mathrm{PBE}+\mathrm{vdW}$ surf method at high-order lithiation stages for $\mathrm{Ti}_{2} \mathrm{CF}_{2}$ systems. While for NG systems, the energy difference from two methods is slight. Our work deepens the understanding of the anchoring mechanism, and provides a more accurate criterion for screening anchoring materials to control shuttle effect and to design more reliable cathode in $\mathrm{Li}-\mathrm{S}$ batteries.

\section{METHODS}

Computational details

Our density functional theory (DFT) calculations were performed using the Fritz-Haber-Institute ab initio molecular simulations (FHI-aims) package ${ }^{45}$ and the Vienna Ab initio Simulation Package (VASP) code. ${ }^{46,47}$ The geometric optimization and the calculations of the charge transfer were performed within the generalized gradient approximation (GGA) of the Perdew-Burke-Ernzerhof (PBE) as implemented in VASP. For geometric optimizations, all atomic positions were relaxed until the maximal forces per atom were less than $0.03 \mathrm{eV} / \AA \AA$ and energy difference was smaller than $10^{-5} \mathrm{eV}$. The plane-wave cutoff energy was set to $450 \mathrm{eV}$. A $3 \times 3$ supercell of monolayer $\mathrm{FeS}\left(\mathrm{SnS}_{2}\right)$, a $5 \times 5$ supercell of $\mathrm{Ti}_{2} \mathrm{CF}_{2}$ with $5 \times 5 \times 1$ 
$\Gamma$-centered $k$-mesh, ${ }^{48}$ and a $7 \times 7$ supercell of NG with $3 \times 3 \times 1 \Gamma$-centered $\mathrm{k}$-mesh were employed to model the adsorption systems. The distance of vacuum region was set to $20 \AA$ along the z-direction (vertical to the interface) to avoid the interaction between adjacent layers. ${ }^{49}$

The FHI-aims code was applied to calculate density of states and energies of all configurations. We used the "tight" settings for numerical atom-centered orbital basis sets. The "tier2" standard basis was utilized for $\mathrm{F}, \mathrm{O}, \mathrm{H}, \mathrm{N}, \mathrm{Li}$ and $\mathrm{C}$, and "tier1" for $\mathrm{S}, \mathrm{Fe}, \mathrm{Ti}$, and $\mathrm{Sn}$. The convergence criteria of $10^{-5}$ electrons per unit volume for the charge density and $10^{-6} \mathrm{eV}$ for the total energy of the systems were used.

\section{Van der Waals inclusive methods}

For the description of the van der Waals (vdW) interactions we applied two methods in DFT calculations. The PBE $+v \mathrm{dW}^{\text {surf }}$ method $^{50}$ were employed to account for the pairwise van der Waals (vdW) interactions. The PBE + $\mathrm{vdW}^{\text {surf }}$ total energy includes the dispersion energy by considering a sum of $-C_{6}^{a b} R_{a b}^{-6}$ terms, where $R_{\mathrm{ab}}$ is the distance between atoms $a$ and $b$, the $C_{6}$ coefficients and vdW radii (vdW parameters) are determined by the collective screening of the substrate electrons, which are captured by mean of the $v_{d W} W^{\text {surf }}$ method. We employed the PBE + MBD method to describe the many-body dispersion (MBD) effect. The MBD method computes the correlation energy of long-range electrostatic screening through the coupled harmonic oscillator model Hamiltonian, and considers dipolar vdW interactions to all orders (many-body effect) in perturbation theory beyond the vdW ${ }^{\text {surf }}$ method.

\section{DATA AVAILABILITY}

The data supporting the findings of this study are available from the corresponding author on reasonable request.

Received: 27 July 2019; Accepted: 3 January 2020; Published online: 30 January 2020

\section{REFERENCES}

1. Crabtree, G. Perspective The energy-storage revolution. Nature 526, S92 (2015).

2. Manthiram, A., Chung, S. H. \& Zu, C. Lithium-sulfur batteries: progress and prospects. Adv. Mater. 27, 1980-2006 (2015).

3. Bruce, P. G., Freunberger, S. A., Hardwick, L. J. \& Tarascon, J. M. Li-O $\mathrm{O}_{2}$ and Li-S batteries with high energy storage. Nat. Mater. 11, 19-29 (2011).

4. Yang, Y., Zheng, G. \& Cui, Y. Nanostructured sulfur cathodes. Chem. Soc. Rev. 42 3018-3032 (2013).

5. Evers, S. \& Nazar, L. F. New approaches for high energy density lithium-sulfur battery cathodes. Acc. Chem. Res. 46, 1135-1143 (2013).

6. Liang, Z., Qu, C., Guo, W., Zou, R. \& Xu, Q. Pristine metal-organic frameworks and their composites for energy storage and conversion. Adv. Mater. 30, e1702891 (2018).

7. Chung, W. J. et al. The use of elemental sulfur as an alternative feedstock for polymeric materials. Nat. Chem. 5, 518-524 (2013).

8. Siczek, K. J. Next-generation Batteries with Sulfur Cathodes. (Academic Press, 2019).

9. Manthiram, A., Fu, Y. \& Su, Y.-S. Challenges and prospects of lithium-sulfur batteries. Acc. Chem. Res. 46, 1125-1134 (2013).

10. Ji, X., Lee, K. T. \& Nazar, L. F. A highly ordered nanostructured carbon-sulphur cathode for lithium-sulphur batteries. Nat. Mater. 8, 500-506 (2009).

11. Song, J. et al. Nitrogen-doped mesoporous carbon promoted chemical adsorption of sulfur and fabrication of high-areal-capacity sulfur cathode with exceptional cycling stability for lithium-sulfur batteries. Adv. Funct. Mater. 24, 1243-1250 (2014).

12. Tao, $X$. et al. Balancing surface adsorption and diffusion of lithium-polysulfides on nonconductive oxides for lithium-sulfur battery design. Nat. Commun. 7, 11203 (2016).

13. Li, Z. et al. A sulfur host based on titanium monoxide@carbon hollow spheres for advanced lithium-sulfur batteries. Nat. Commun. 7, 13065 (2016).

14. Hu, L. et al. A highly efficient double-hierarchical sulfur host for advanced lithiumsulfur batteries. Chem. Sci. 9, 666-675 (2018).

15. $\mathrm{Li}, \mathrm{C}$. et al. Titanium nitride hollow nanospheres with strong lithium polysulfide chemisorption as sulfur hosts for advanced lithium-sulfur batteries. Nano Res. 11, 4302-4312 (2018).

16. Naguib, M. et al. Two-dimensional transition metal carbides. ACS Nano 6, 1322-1331 (2012).
17. Xie, Y. et al. Role of surface structure on Li-ion energy storage capacity of twodimensional transition-metal carbides. J. Am. Chem. Soc. 136, 6385-6394 (2014).

18. Mashtalir, O., Lukatskaya, M. R., Zhao, M. Q., Barsoum, M. W. \& Gogotsi, Y. Amineassisted delamination of $\mathrm{Nb}_{2} \mathrm{C}$ MXene for Li-ion energy storage devices. Adv. Mater. 27, 3501-3506 (2015).

19. Luo, J. et al. Pillared structure design of mxene with ultralarge interlayer spacing for high-performance lithium-ion capacitors. ACS Nano 11, $2459-2469$ (2017).

20. Xiong, D., Li, X., Bai, Z. \& Lu, S. Recent advances in layered $\mathrm{Ti}_{3} \mathrm{C}_{2} \mathrm{~T}_{\mathrm{x}}$ MXene for electrochemical energy storage. Small 14, e1703419 (2018).

21. Anasori, B., Lukatskaya, M. R. \& Gogotsi, Y. 2D metal carbides and nitrides (MXenes) for energy storage. Nat. Rev. Mater. 2, 16098 (2017).

22. Song, J. et al. Immobilizing polysulfides with mxene-functionalized separators for stable lithium-sulfur batteries. ACS Appl. Mater. Interfaces 8, 29427-29433 (2016).

23. Zhou, F. et al. Low cost metal carbide nanocrystals as binding and electrocatalytic sites for high performance Li-S batteries. Nano. Lett. 18, 1035-1043 (2018).

24. Bao, W. et al. Facile synthesis of crumpled nitrogen-doped MXene nanosheets as a new sulfur host for lithium-sulfur batteries. Adv. Energy Mater. 8, 1702485 (2018).

25. Wang, H., Zhang, W., Xu, J. \& Guo, Z. Advances in polar materials for lithium-sulfur batteries. Adv. Funct. Mater. 28, 1707520 (2018).

26. Hou, T. Z. et al. Design principles for heteroatom-doped nanocarbon to achieve strong anchoring of polysulfides for lithium-sulfur batteries. Small 12, 3283-3291 (2016).

27. Zhang, Q. et al. Understanding the anchoring effect of two-dimensional layered materials for lithium-sulfur batteries. Nano Lett. 15, 3780-3786 (2015).

28. Zhou, G. et al. Catalytic oxidation of $\mathrm{Li}_{2} \mathrm{~S}$ on the surface of metal sulfides for $\mathrm{Li}$-S batteries. Proc. Natl Acad. Sci. USA 114, 840-845 (2017).

29. Sim, E. S., Yi, G. S., Je, M., Lee, Y. \& Chung, Y.-C. Understanding the anchoring behavior of titanium carbide-based MXenes depending on the functional group in Li-S batteries: A density functional theory study. J. Power Sources 342, 64-69 (2017).

30. Yin, L.-C. et al. Understanding the interactions between lithium polysulfides and $\mathrm{N}$-doped graphene using density functional theory calculations. Nano Energy 25, 203-210 (2016).

31. Brandenburg, J. G. et al. Physisorption of water on graphene: subchemical accuracy from many-body electronic structure methods. J. Phys. Chem. Lett. 10, 358-368 (2019).

32. Yang, S., Jiang, Y., Li, S. \& Liu, W. Many-body dispersion effects on the binding of TCNQ and F4-TCNQ with graphene. Carbon 111, 513-518 (2017).

33. Maurer, R. J., Ruiz, V. G. \& Tkatchenko, A. Many-body dispersion effects in the binding of adsorbates on metal surfaces. J. Chem. Phys. 143, 102808 (2015).

34. Liu, W. et al. Quantitative prediction of molecular adsorption: structure and binding of benzene on coinage metals. Phys. Rev. Lett. 115, 036104 (2015).

35. Qiu, Y. et al. High-rate, ultralong cycle-life lithium/sulfur batteries enabled by nitrogen-doped graphene. Nano Lett. 14, 4821-4827 (2014).

36. Zhang, $H$. et al. Designing flexible 2D transition metal carbides with strain-controllable lithium storage. Proc. Natl Acad. Sci. USA 114, E11082-E11091 (2017).

37. Fu, Z. et al. Mechanistic quantification of thermodynamic stability and mechanical strength for two-dimensional transition-metal carbides. J. Phys. Chem. C 122 4710-4722 (2018).

38. Kamphaus, E. P. \& Balbuena, P. B. First-principles investigation of lithium polysulfide structure and behavior in solution. J. Phys. Chem. C. 121, 21105-21117 (2017).

39. Ambrosetti, A., Ferri, N., DiStasio, R. A. \& Tkatchenko, A. Wavelike charge density fluctuations and van der Waals interactions at the nanoscale. Science 351, 1171 (2016).

40. Tkatchenko, A., DiStasio, R. A. Jr., Car, R. \& Scheffler, M. Accurate and efficient method for many-body van der Waals interactions. Phys. Rev. Lett. 108, 236402 (2012).

41. Bader, R. Atoms in Molecules-A Quantum Theory. (Oxford University, 1990).

42. Wang, D. et al. A general atomic surface modification strategy for improving anchoring and electrocatalysis behavior of $\mathrm{Ti}_{3} \mathrm{C}_{2} \mathrm{~T}_{2} \mathrm{MXene}$ in lithium-sulfur batteries. ACS Nano 13, 11078-11086 (2019).

43. Chen, $\mathrm{S}$. et al. Surface electrochemical stability and strain-tunable lithium storage of highly flexible 2D transition metal carbides. Adv. Funct. Mater. 28, 1804867 (2018).

44. Ibragimova, R., Puska, M. J. \& Komsa, H. P. pH-dependent distribution of functional groups on titanium-based MXenes. ACS Nano 13, 9171-9181 (2019).

45. Blum, V. et al. Ab initio molecular simulations with numeric atom-centered orbitals. Comput. Phys. Commun. 180, 2175-2196 (2009).

46. Kresse, G. \& Furthmüller, J. Efficiency of ab-initio total energy calculations for metals and semiconductors using a plane-wave basis set. Comput. Mater. Sci. 6 , 15-50 (1996). 
47. Kresse, G. \& Furthmüller, J. Efficient iterative schemes for ab initio totalenergy calculations using a plane-wave basis set. Phys. Rev. B 54, 11169-11186 (1996).

48. Monkhorst, H. J. \& Pack, J. D. Special points for Brillouin-zone integrations. Phys. Rev. B 13, 5188-5192 (1976).

49. Liu, W., Tkatchenko, A. \& Scheffler, M. Modeling adsorption and reactions of organic molecules at metal surfaces. Acc. Chem. Res. 47, 3369-3377 (2014).

50. Ruiz, V. G., Liu, W., Zojer, E., Scheffler, M. \& Tkatchenko, A. Density-Functional Theory with Screened van der Waals Interactions for the Modeling of Hybrid Inorganic-Organic Systems. Phys. Rev. Lett. 108, 146103 (2012).

\section{ACKNOWLEDGEMENTS}

We acknowledge supports from the NSF of China (51602155, 51722102, 21773120), the NSF of Jiangsu Province (BK20180448), the Fundamental Research Funds for the Central Universities (30918011340, 30919011405, 30917011201) and Jiangsu Key Laboratory of Advanced Micro\&Nano Materials and Technology.

\section{AUTHOR CONTRIBUTIONS}

S.L. and W.L. directed and designed the whole research. M.F. did all calculations and wrote the manuscript. All authors discussed the results and provided inputs to the manuscript.

\section{COMPETING INTERESTS}

The authors declare no competing interests.

\section{ADDITIONAL INFORMATION}

Supplementary information is available for this paper at https://doi.org/10.1038/ s41524-020-0273-1.

Correspondence and requests for materials should be addressed to S.L. or W.L.

Reprints and permission information is available at http://www.nature.com/ reprints

Publisher's note Springer Nature remains neutral with regard to jurisdictional claims in published maps and institutional affiliations.

Open Access This article is licensed under a Creative Commons Attribution 4.0 International License, which permits use, sharing, adaptation, distribution and reproduction in any medium or format, as long as you give appropriate credit to the original author(s) and the source, provide a link to the Creative Commons license, and indicate if changes were made. The images or other third party material in this article are included in the article's Creative Commons license, unless indicated otherwise in a credit line to the material. If material is not included in the article's Creative Commons license and your intended use is not permitted by statutory regulation or exceeds the permitted use, you will need to obtain permission directly from the copyright holder. To view a copy of this license, visit http://creativecommons. org/licenses/by/4.0/.

(c) The Author(s) 2020 